\title{
Exotic circuit elements from zero-modes in hybrid superconductor/quantum Hall systems
}

\author{
David J. Clarke, ${ }^{1}$ Jason Alicea, ${ }^{1}$ and Kirill Shtengel ${ }^{2}$ \\ ${ }^{1}$ Department of Physics, California Institute of Technology, Pasadena, CA 91125, USA \\ ${ }^{2}$ Department of Physics and Astronomy, University of California, Riverside, CA 92521, USA
}

\begin{abstract}
Heterostructures formed by quantum Hall systems and superconductors have recently been shown to support widely coveted Majorana fermion zero-modes and still more exotic 'parafermionic' generalizations. Here we establish that probing such zero-modes using quantum Hall edge states yields non-local transport signatures that pave the way towards a variety of novel circuit elements. In particular, we demonstrate quite generally that at low energies the zero-modes convert chirally moving quasiparticles into oppositely charged quasiholes propagating in the same direction - that is, they swap the sign of the chiral edge currents. One may then construct new and potentially useful circuit elements using this 'perfect Andreev conversion' process, including superconducting current and voltage mirrors as well as transistors for fractional charge currents. Characterization of these circuit elements should provide striking evidence of the zero-mode physics.
\end{abstract}

Introduction. Non-Abelian anyons provide a fascinating illustration of Anderson's 'more is different' paradigm [1]. These quasiparticles, which emerge from interacting collections of ordinary bosons and fermions, produce a groundstate degeneracy that scales exponentially with the number of anyons present in the host system. Moreover, braiding the anyons around one another noncommutatively rotates the system's quantum state within this degenerate manifold. These remarkable properties have led to great interest in non-Abelian anyons for use in fault-tolerant quantum information processing devices [2]. Our goal here is to propose another application of non-Abelian anyons, namely the construction of unusual circuit elements such as transistors for fractional charge, current/voltage mirrors, and flux-based capacitors.

As a primer, let us first consider a one-dimensional (1D) topological superconductor [3], obtained when an oddchannel wire acquires a bulk pairing gap. Suppose that a 1D superconductor breaks up into alternating topological and trivial domains as in Fig. 1 (a) (e.g., by varying the number of channels spatially along the wire). Here the endpoints of the topological regions realize 'Ising' non-Abelian anyons, which bind Majorana zero-modes that encode a twofold ground-state degeneracy per topological segment. Physically, the degeneracy reflects the fact that each topological domain can switch its fermion parity without affecting the energy density-contrary to conventional superconductors. Numerous sources of 1D topological superconductivity have been proposed, which involve coupling a bulk superconductor to systems such as two-dimensional (2D) topological insulator edges [4], spin-orbit-coupled nanowires [5, 6], magnetic-atom chains [7, 8], or counterpropagating sets of integer quantum Hall edge modes [9, 10] (for reviews see Refs. [11-14]).

Among these platforms, the integer quantum Hall architecture most naturally generalizes to fractionalized setups that harbor richer phenomena stemming from the interplay between superconductivity and strong correlations. Consider, for instance, a 'wire' synthesized from counterpropagating fractional quantum Hall edge states separated by a narrow trench [see Fig. 1 b) for an example at filling $\nu=2 / 3$ ]. This 'wire' can acquire a gap either through electron backscattering across the trench or via Cooper pairing. Because (a) 1D Superconductor

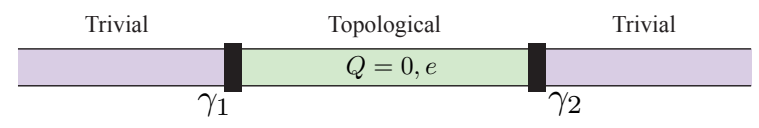

(b) FQH edge-state 'wire'

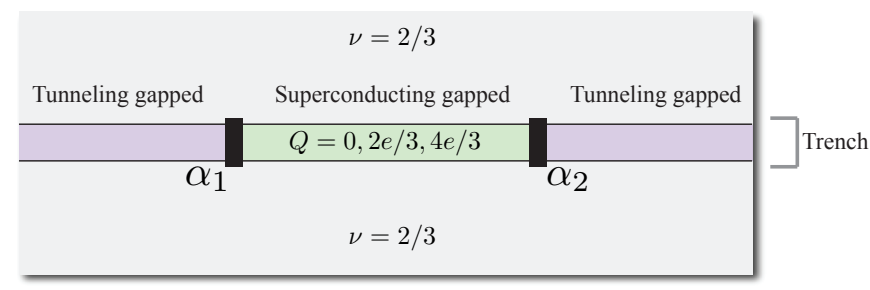

FIG. 1: (a) Majorana zero-modes $\gamma_{j}$ bind to domain walls separating topological and trivial segments of a 1D superconducting wire. (b) 'Parafermion' zero-modes $\alpha_{j}$, which represent $\mathbb{Z}_{3}$ generalized Majorana modes, localize between segments of a trench in a $\nu=2 / 3$ quantum Hall state gapped by tunneling and Cooper pairing. In both cases the zero-modes encode a degeneracy among states with different charge $Q(\bmod 2 e)$ on the central region, as labeled in the figure.

the edge states support fractionally charged excitations, the ends of pairing-gapped regions correspond to exotic nonAbelian anyons binding generalizations of Majorana zeromodes [9, 10, 15]. These parafermionic zero-modes [16] encode a larger ground-state degeneracy compared to the usual Majorana case, since here each superconducting-gapped region can acquire fractional charge without changing its energy density. Similar effects may arise in other fractionalized setups [17-21], including quantum Hall bilayers in which interlayer tunneling plays the role of Cooper pairing [22, 23].

In this paper we predict novel non-local transport signatures of Majorana and parafermionic zero-modes in quantum Hall/superconductor hybrids that, in turn, provide a foundation for the unusual circuit elements mentioned above. The experiments we propose relate closely to the 'zero-bias anomaly' arising when a single-channel normal lead probes a Majorana zero-mode in a 1D topological superconductor [2432]. In such a setup —sketched in Fig. 2(a) — the Majorana 
mode is predicted to mediate perfect Andreev reflection as temperature $T$ and bias voltage $V$ approach zero. That is, in this asymptotic limit an incoming electron from the normal lead reflects off of the topological superconductor as a hole with unit probability, yielding a quantized zero-bias conductance of $2 e^{2} / h$. Importantly, this is twice the conductance of an ideal single-channel wire, with the factor of two arising because of the added contribution of the reflected hole.

We show that quantum Hall/superconductor hybrids yield an interesting variation on this transport anomaly, in particular when the native edge states serve as a lead that probes zeromodes generated in such setups. The basic transport architecture appears in Fig. 2(b) and contains two new features compared to the 1D topological superconductor problem. First, in the fractional quantum Hall case the (parafermionic) zeromode at the outer trench edge mediates perfect conversion of incoming quasiparticles carrying fractional charge $e^{*}$ into $-e^{*}$ quasiholes as $T, V \rightarrow 0$, thereby transmitting charge $2 e^{*}$ into the superconductor. Such events are possible since the pairing-gapped trench can resonantly absorb fractional charge due to the ground-state degeneracy. (Ref. [23] briefly explored an analogous transport phenomenon in a bilayer setup.) Second-and more importantly-if the (ungapped) quantum Hall edge used as a lead supports purely chiral charge excitations, the outgoing quasihole continues in the same direction around the edge as Fig. 2(b) illustrates. We refer to this process as Andreev conversion to distinguish it from standard Andreev reflection in which the hole retraces the incoming particle's path [43]. The superconductor and quantum Hall edges thus form a trijunction in which the current and voltage in each leg exhibits a strong dynamical constraint. When multiple superconducting trenches are immersed in the same quantum Hall fluid, this dynamical constraint underlies various non-local transport anomalies. In what follows we theoretically establish the perfect Andreev conversion noted above under rather general circumstances and then discuss several novel circuit elements that follow.

Perfect Andreev conversion. One can access the transport phenomena we describe using many different quantum Hall phases. The conceptually simplest corresponds to a $\nu=1$ integer quantum Hall state, though coupling to superconductivity in this case appears non-trivial due to spin polarization. Alternatively, the $\nu=1$ edge mode can arise from the magnetically gapped surface of a 3D topological insulator [33, 34]. In this realization one can utilize ordinary $s$-wave superconductors; moreover, orbital magnetic fields are not required. Quantum Hall phases with multiple edge channels also suffice. We will simply require that the edge supports a single, chiral charge mode that at low energies decouples from all other neutral modes. This decoupling occurs, e.g., for hierarchical states at $\nu=n /(2 n p+1)$ ( $n$ and $p$ are integers) due to disorder as shown by Kane and Fisher [35]. Among this series the spin-unpolarized $\nu=2 / 3$ state is particularly advantageous, in part because here too one can induce pairing via an $s$-wave superconductor. Another virtue is that the instabilities leading to the zero-modes occur at weak coupling (a)

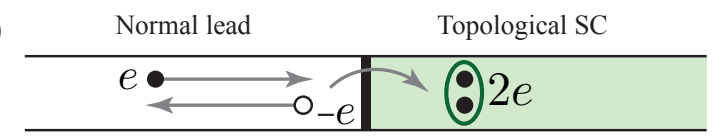

(b)

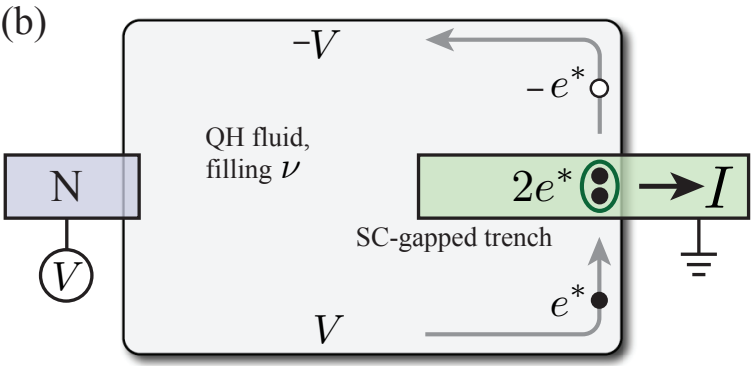

FIG. 2: (a) Electrons injected from a normal lead towards a 1D topological superconductor perfectly Andreev reflect into holes due to coupling with a Majorana zero-mode at the junction. (b) The zero-mode in a pairing-gapped trench of a quantum Hall fluid similarly mediates 'perfect Andreev conversion', transforming incoming quasiparticles from the edge into outgoing quasiholes. In either case, the superconductor absorbs the excess charge.

for a range of realistic parameters. We expound on this important technical point in the Supplementary Information, which explores the $\nu=2 / 3$ case in greater detail.

Suppose that we etch a trench into a quantum Hall system and then fill the void with a superconductor as shown in Fig. 2(b). Provided the trench width does not exceed the superconductor's coherence length, the proximity effect can then gap out the adjacent edge modes through Cooper pairing. As noted earlier one thereby creates zero-modes at the ends of the trench that encode a ground-state degeneracy for the intervening pairing-gapped region. We will now explore transport resulting when the gapless chiral charge mode impinging on the trench hybridizes with one of these zero-modes.

The key physical mechanism is that at low energies the gapped trench imposes certain boundary conditions that relate incoming and outgoing quasiparticles from the adjacent gapless edge. Consider first the limit in which the gapless edge state completely decouples from the superconducting trench. Concretely, one could envision adding near the boundary a small tunneling-gapped region as in Fig. 1 (b) to block coupling to the zero-mode. In this case incoming quasiparticles continue along the edge uninterrupted by the trench, and the action governing the charge mode of interest is simply that of an unperturbed edge [36]:

$$
S_{\text {charge }}=\frac{-1}{4 \pi \nu} \int \mathrm{d} x \mathrm{~d} t \partial_{x} \phi\left(\partial_{t}+v \partial_{x}\right) \phi,
$$

with $\phi$ a field that determines the total edge charge density through $\rho=e \partial_{x} \phi /(2 \pi)$. Commutation relations implicit in the action imply that $e^{i \phi}$ is an operator that adds charge $e^{*}=\nu e$. Throughout we assume that this charge mode decouples from all neutral modes - should any exist. Equation (1) describes a fixed point for the edge at which particles undergo 'perfect normal transmission' upon hitting the trench. 
Next we incorporate weak coupling to the zero-mode nearest to the edge, which allows charge $e^{*}$ to pass between the gapless edge and the pairing-gapped region. Such processes perturb the above fixed-point action with a term

$$
\delta S_{\text {zero-mode }}=\Gamma \int \mathrm{d} t\left[e^{i \Phi} e^{-i \phi\left(x=x_{0}, t\right)}+\text { H.c. }\right],
$$

where $x_{0}$ is the position where the trench and gapless charge mode intersect, $\Gamma$ denotes the bare coupling strength, and $e^{i \Phi}$ is an operator that cycles the charge on the pairing-gapped region of the trench by $e^{*}(\bmod 2 e)$. Under renormalization $\Gamma$ flows according to $\partial_{\ell} \Gamma=(\Delta-1) \Gamma$, where $\ell$ is a logarithmic rescaling factor and $\Delta=\nu / 2$ is the scaling dimension of the tunneling operator in Eq. (2). For $\nu<2$-to which we specialize hereafter-hybridization with the zero-mode thus constitutes a relevant perturbation that destabilizes the perfect normal transmission fixed point.

The system then flows to a different fixed point at which coupling to the zero-mode imposes nontrivial boundary conditions on the gapless charge mode at $x=x_{0}$. This boundary condition can be expressed as

$$
\phi_{\text {out }}=2 \Phi-\phi_{\text {in }}
$$

with $\phi_{\text {out } / \text { in }} \equiv \phi\left(x=x_{0} \pm 0^{+}\right)$denoting gapless chargesector fields evaluated just above and below the trench. Equation (3) causes incoming charge- $e^{*}$ quasiparticles $\left(e^{i \phi}\right)$ added relative to the superconductor's potential to continue as outgoing $-e^{*}$ quasiholes $\left(e^{-i \phi}\right)$, with the pairing-gapped trench absorbing the deficit charge $2 e^{*}\left(e^{2 i \Phi}\right)$. This is precisely the Andreev conversion process described earlier. There is, however, a competing effect, whereby electrons tunnel directly past the trench. We now address the relevance of these processes at the 'perfect Andreev conversion' fixed point defined by the boundary condition in Eq. (3).

Any perturbation that tunnels an electron past the trench takes the form

$$
\begin{aligned}
\delta S_{\text {tunneling }} & =\lambda \int \mathrm{d} t\left[e^{i \phi_{\mathrm{in}} / \nu-i \phi_{\text {out }} / \nu} \mathcal{O}_{n}+\text { H.c. }\right] \\
& \rightarrow \lambda \int \mathrm{d} t\left[e^{2 i\left(\phi_{\mathrm{in}}-\Phi\right) / \nu} \mathcal{O}_{n}+\text { H.c. }\right]
\end{aligned}
$$

where $\lambda$ is the coupling strength and $\mathcal{O}_{n}$ contains possible neutral parts of the electron tunneling operator (e.g., operators that transfer spin). In the second line we used Eq. (3) to eliminate $\phi_{\text {out }}$. The scaling dimension of the above electron tunneling term reads $\Delta_{e}=(2 / \nu)+\Delta_{n}$, with $\Delta_{n}$ the scaling dimension of $\mathcal{O}_{n}$. Consequently, any operator that transfers electron charge across the trench has $\Delta_{e}>2 / \nu$ and is hence irrelevant for $\nu<2$-implying stability of the perfect Andreev conversion fixed point in the regime of interest [44]. Numerous interesting consequences for transport follow from this general result.

Doubled Hall voltage. Consider again the setup of Fig. 2(b), where a normal electrode at potential $V$ injects charge along the lower quantum Hall edge towards a grounded superconducting trench. We temporarily assume negligible contact resistances; the lower edge is then also at potential $V$. For 'small' $V$ (quantified below), injected charge undergoes perfect Andreev conversion at the trench. Thus the conductance $G$ doubles compared to a standard two-terminal measurement-i.e., $G=2 \sigma_{\mathrm{H}}$, where $\sigma_{\mathrm{H}}=\nu e^{2} / h$ is the Hall conductance. For $\nu=1$ the physics essentially maps to the 1D topological superconductor case described earlier, modulo the spatial separation of incoming and outgoing modes.

The potential on the upper quantum Hall edge, where the outgoing Andreev-converted carriers flow, follows from current conservation. More precisely, since the same current $I=G V$ passing through the superconductor traverses the quantum Hall fluid, the current must additionally satisfy $I=$ $\sigma_{\mathrm{H}} V_{H}\left(V_{H}\right.$ is the Hall voltage). Consistency requires that the Hall voltage, like the conductance, is also doubled compared to the case where both source and drain are normal electrodes. That is, the potential on the upper edge is $-V$, opposite that of the lower edge. This implies that the Andreev-converted carriers do not equilibrate with the superconductor; rather equilibration occurs at the normal electrode in Fig. 2(b).

Consider next the more general situation where the potential for the superconductor is $V_{\mathrm{SC}}$ and that of the incoming/outgoing quantum Hall edges is $V_{\text {in/out }}$. Due to the doubled conductance the current flowing out of the superconductor is $I=2 \sigma_{\mathrm{H}}\left(V_{\mathrm{in}}-V_{\mathrm{SC}}\right)$, while the (same) current crossing the Hall fluid obeys $I=\sigma_{\mathrm{H}}\left(V_{\text {in }}-V_{\text {out }}\right)$. It follows that

$$
V_{\text {out }}=2 V_{\mathrm{SC}}-V_{\text {in }}
$$

which we will frequently employ in the forthcoming discussion. These results apply when temperature and the superconductor/quantum Hall voltage differences are $(i)$ small compared to the zero-mode hybridization energy $\Gamma$ [recall Eq. (2)] and $(i i)$ sufficiently small that the irrelevant electron tunneling terms [Eq. 44] remain inoperative; this is the regime where perfect Andreev conversion holds. Note also that imperfect superconducting contact will decrease the voltage drop compared to Eq. 55. In general, $V_{\text {out }}=V_{\text {in }}+\eta\left(V_{\mathrm{SC}}-V_{\text {in }}\right)$, where the quality factor $\eta$ ranges between 0 (no contact) and 2 (ideal superconducting contact). We stress that if both electrodes were normal, $\eta$ could never exceed 1 . Unless otherwise stated, we will assume ideal superconducting contacts in the devices described below to emphasize their unusual behavior.

Transistor for fractional charge. Figure 3 illustrates a simple example of non-local effects resulting when multiple superconducting trenches appear in the quantum Hall system. Here, all superconductors are set to the same potential. Unlike in the Josephson effect, their relative phases are inconsequential - we assume that any notion of phase coherence is lost along the edges connecting the leads. The charge, however, is conserved in such transport and, as a consequence, in Fig. 3. a) - where the number of superconductors is evencurrent can only flow from one superconductor to another if it flows in all four simultaneously, with relative orientations specified in the figure. Sending current from the left to bottom 

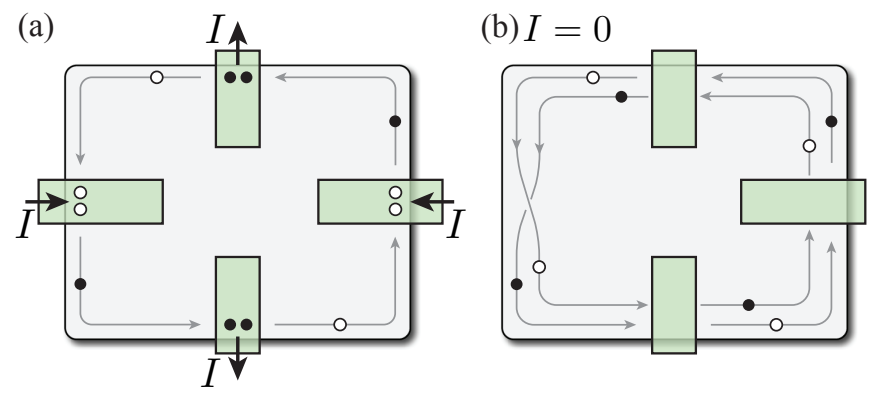

FIG. 3: (a) Quantum Hall fluid with four pairing-gapped trenches generated by equipotential superconductors. If each superconductor induces perfect Andreev conversion, the same current $I$ must flow through all four superconductors, with relative orientation indicated by the large arrows. (b) If an odd number of equipotential superconductors enter the quantum Hall fluid, the system can no longer carry dc current, as the charge added to the superconductors due to Andreev conversion time-averages to zero.
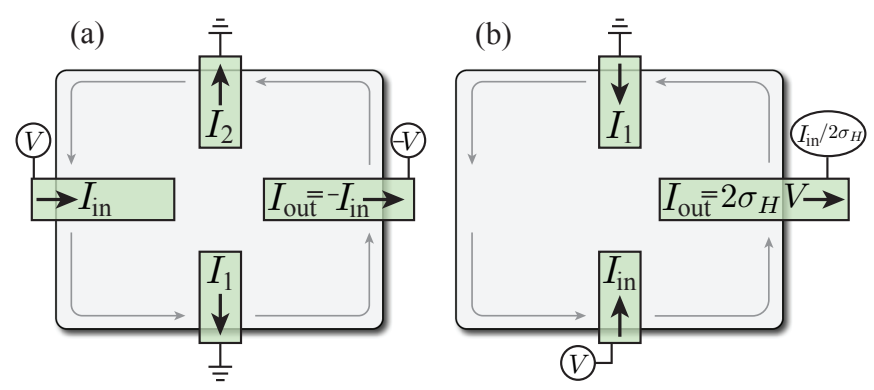

FIG. 4: (a) Current/voltage mirror and (b) current/voltage swap circuit elements generated from perfect Andreev conversion.

superconductors, for instance, necessarily yields the same current flow from right to top, a behavior reminiscent of the nonlocal transport mediated by excitons in bilayer quantum Hall systems [37]. The restricted current arises because $(i)$ quasiparticles undergo perfect Andreev conversion at each trench and (ii) a given superconductor receives the same charge under subsequent 'round trips' along the edge. By contrast, with an odd number of equipotential superconductors, as in Fig. 3 b), (direct) current can simply not flow. This becomes evident upon tracing the path of a single quasiparticle around the edge. On the first round trip, the quasiparticle deposits charge on each superconductor due to Andreev conversion, but on the next pass removes these same charges because the number of superconductors is odd. Figure 3 illustrates the relevant quasiparticle processes in both cases. One can utilize this even/odd effect to create a transistor for fractional charge by using gates to controllably isolate one of the superconductors from the rest of the system.

Voltage/Current Mirror. Suppose we have a device with four superconducting trenches [as in Fig. 3(a)] but now allow their potentials to vary. The constraints imposed by Eq. (5), together with the doubled conductance relating voltage to current, leave the system with only three remaining degrees of freedom. The first corresponds to current flow in the pattern

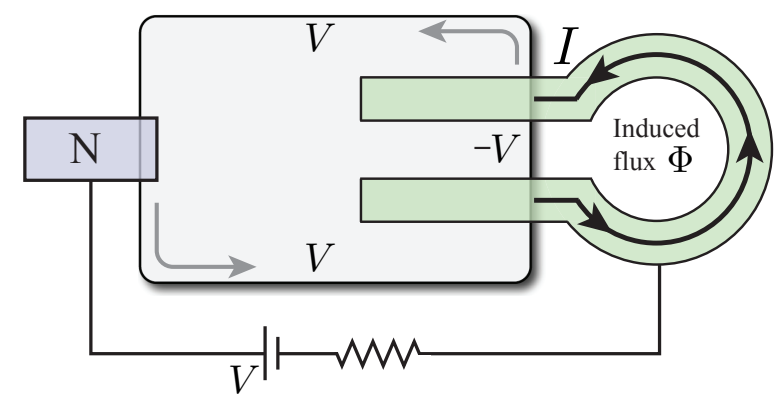

FIG. 5: A voltage $V$ applied to the normal electrode on the left generates current in the superconducting loop on the right. The induced flux is proportional to $V$-hence the circuit acts as a 'flux capacitor'. Unlike a standard inductor current need not flow through the battery.

shown by Fig. 3(a). A second degree of freedom appears in Fig. 4(a), where we ground the upper and lower superconductors while raising the voltage of the left superconductor (the control). The voltage of the remaining superconductor (the output) necessarily goes down, mirroring the change. As in Fig. 3 a), the current carried away by the output is opposite that flowing into the control; excess current flows to ground via the top and bottom superconductors. We are left, then, with a device that reverses both the voltage and the current flow from input to output. One can access the final degree of freedom by changing the relative voltage of the two grounded superconductors. The response to such a change may be interpreted as a superposition of Fig. 4 (a) with the same setup rotated by $90^{\circ}$.

Voltage/Current Swap. In a system with three ideal equipotential superconducting trenches, we have already shown that no current can flow [recall Fig. 3 (b)]. However, suppose that —as in Fig. 4 b) — we ground only the top superconductor and set the current and voltage on the bottom (control) superconductor. The third superconductor functions as the output. In this case the constraints of Eq. (5) lead to the unusual result that the two independent information channels (for a superconductor), current and voltage, are swapped at the output relative to the input. For instance, if the current flowing into the control terminal is $I_{\text {in }}$ then the voltage at the output is $I_{\text {in }} /\left(2 \sigma_{\mathrm{H}}\right)$; likewise, if the control terminal voltage is $V$, the output current is $I_{\text {out }}=2 \sigma_{\mathrm{H}} V$. (Excess current again flows to ground.)

Superconducting Flux-based Capacitor. Consider next Fig. 4(b) in the limit where the right superconductor is also held to ground. In this case the input current $I_{\text {in }}$ must clearly vanish. Nevertheless, the voltage $V$ on the bottom control superconductor induces a current $2 \sigma_{\mathrm{H}} V$ flowing from the top to the right superconducting contacts, even though the latter remain at the same potential. Note that this is consistent with Fig. 3 b) since in the limit $V=0$ all currents vanish. This effect persists for larger odd numbers of superconductors as well, in which case current of magnitude $2 \sigma_{\mathrm{H}} V$ flows in all grounded terminals, with a sign alternating from lead to lead.

Finally, let us examine the circuit in Fig. 5, where we use 
analogous physics to create a 'flux capacitor' in which a voltage stores magnetic flux. Here a pair of superconducting trenches connect into a loop on the right side, while a normal electrode on the left is held at potential $V$ relative to the superconductors by a battery. Once again using Eq. (5) to determine the voltages along the circuit, we find that current $I=2 \sigma_{\mathrm{H}} V$ flows around the superconducting loop, generating flux $\Phi \equiv C_{\Phi} V$. The 'flux capacitance' $C_{\Phi}$ is determined by the loop's inductance. We emphasize that unlike an ordinary inductor, no current flows through the power source or resistor at steady state in the ideal $\eta=2$ device. Rather, the applied voltage merely sets the level of circulating current in the superconducting loop.

Discussion. It is worth reiterating that the non-local transport anomalies in the devices proposed above originate from zero-modes bound to non-Abelian defects induced by the superconductors. Characterization of these circuit elements is therefore a natural first step in the pursuit of topological quantum computation with such systems [9, 10, 15, 38]. Another tantalizing application is the construction of low-power logical circuits. These circuits would have an advantage in the control of low-temperature quantum information devices, as they would coexist within the same low-temperature environment. Depending on the implementation, it may also be possible to produce such logical circuits 'on-chip' with quantum information implementations already based on 2D-electron gas and/or superconducting elements. These applications likely extend to alternative setups as well. The topological-insulatorbased interferometers proposed in Refs. [33, 34] yield a related mechanism for perfect Andreev conversion, and thus should also form the backbone of nontrivial circuit elements. Quantum Hall bilayers (without superconductivity) provide another promising venue. Novel dc transformers were proposed in this context a decade ago [39], and given that a bilayer variant of Andreev conversion is already theoretically established [23] other interesting elements are likely also possible.

Acknowledgements. We are indebted to J. P. Eisenstein, M. P. A. Fisher, C. Nayak and A. Stern for numerous enlightening discussions. We also acknowledge funding from the NSF through grants DMR-1341822 (D. J. C. \& J. A.) and DMR-0748925 (K. S.); the Alfred P. Sloan Foundation (J. A.); the DARPA QuEST program (K. S.); and the Caltech Institute for Quantum Information and Matter, an NSF Physics Frontiers Center with support of the Gordon and Betty Moore Foundation.

[1] P. W. Anderson, Science 177, 393 (1972).

[2] C. Nayak, S. H. Simon, A. Stern, M. Freedman, and S. Das Sarma, Rev. Mod. Phys. 80, 1083 (2008), arXiv:0707.1889.

[3] A. Y. Kitaev, Phys.-Usp. 44, 131 (2001), cond-mat/0010440.

[4] L. Fu and C. L. Kane, Phys. Rev. Lett. 100, 096407 (2008), arXiv:0707.1692.
[5] R. M. Lutchyn, J. D. Sau, and S. Das Sarma, Phys. Rev. Lett. 105, 077001 (2010), arXiv:1002.4033.

[6] Y. Oreg, G. Refael, and F. von Oppen, Phys. Rev. Lett. 105, 177002 (2010), arXiv:1003.1145.

[7] T.-P. Choy, J. M. Edge, A. R. Akhmerov, and C. W. J. Beenakker, Phys. Rev. B 84, 195442 (2011), arXiv:1108.0419.

[8] S. Nadj-Perge, I. K. Drozdov, B. A. Bernevig, and A. Yazdani, Phys. Rev. B 88, 020407 (2013), arXiv:1303.6363.

[9] D. J. Clarke, J. Alicea, and K. Shtengel, Nat. Commun. 4, 1348 (2013), arXiv:1204.5479.

[10] N. H. Lindner, E. Berg, G. Refael, and A. Stern, Phys. Rev. X 2, 041002 (2012), arXiv:1204.5733.

[11] C. W. J. Beenakker, Annu. Rev. Condens. Matter Phys. 4, 113 (2013), arXiv:1112.1950.

[12] J. Alicea, Rep. Prog. Phys. 75, 076501 (2012), arXiv:1202.1293.

[13] M. Leijnse and K. Flensberg, Semicond. Sci. Technol. 27, 124003 (2012), arXiv:1206.1736.

[14] T. D. Stanescu and S. Tewari, J. Phys.: Condens. Matter 25, 233201 (2013), arXiv:1302.5433.

[15] M. Cheng, Phys. Rev. B 86, 195126 (2012), arXiv:1204.6084.

[16] P. Fendley, J. Stat. Mech. p. P11020 (2012), arXiv:1209.0472.

[17] M. Barkeshli and X.-L. Qi, Phys. Rev. X 2, 031013 (2012), arXiv:1112.3311.

[18] A. Vaezi, Phys. Rev. B 87, 035132 (2013), arXiv:1204.6245.

[19] Y. Oreg, E. Sela, and A. Stern (2013), arXiv:1301.7335.

[20] J. Klinovaja and D. Loss (2013), arXiv:1311.3259.

[21] J. Klinovaja and D. Loss (2013), arXiv:1312.1998.

[22] M. Barkeshli, C.-M. Jian, and X.-L. Qi, Phys. Rev. B 87, 045130 (2013), arXiv:1208.4834.

[23] M. Barkeshli and X.-L. Qi (2013), arXiv:1302.2673.

[24] K. Sengupta, I. Žutić, H.-J. Kwon, V. M. Yakovenko, and S. Das Sarma, Phys. Rev. B 63, 144531 (2001), condmat/0010206.

[25] C. J. Bolech and E. Demler, Phys. Rev. Lett. 98, 237002 (2007), cond-mat/0607779.

[26] K. T. Law, P. A. Lee, and T. K. Ng, Phys. Rev. Lett. 103, 237001 (2009), arXiv:0907.1909.

[27] K. Flensberg, Phys. Rev. B 82, 180516 (2010), arXiv:1009.3533.

[28] M. Wimmer, A. R. Akhmerov, J. P. Dahlhaus, and C. W. J. Beenakker, New J. Phys. 13, 053016 (2011), arXiv:1101.5795.

[29] L. Fidkowski, J. Alicea, N. H. Lindner, R. M. Lutchyn, and M. P. A. Fisher, Phys. Rev. B 85, 245121 (2012), arXiv:1203.4818.

[30] C.-H. Lin, J. D. Sau, and S. Das Sarma, Phys. Rev. B 86, 224511 (2012), arXiv:1204.3085.

[31] I. Affleck and D. Giuliano, J. Stat. Mech. 2013, P06011 (2013), arXiv:1305.1888.

[32] R. M. Lutchyn and J. H. Skrabacz, Phys. Rev. B 88, 024511 (2013), arXiv:1302.0289.

[33] L. Fu and C. L. Kane, Phys. Rev. Lett. 102, 216403 (2009), arXiv:0903.2427.

[34] A. R. Akhmerov, J. Nilsson, and C. W. J. Beenakker, Phys. Rev. Lett. 102, 216404 (2009), arXiv:0903.2196.

[35] C. L. Kane and M. P. A. Fisher, Phys. Rev. B 51, 13449 (1995).

[36] X.-G. Wen, Quantum Field Theory of Many-Body Systems, Oxford Graduate Texts (Oxford University Press, Oxford, 2004).

[37] A. D. K. Finck, J. P. Eisenstein, L. N. Pfeiffer, and K. W. West, Phys. Rev. Lett. 106, 236807 (2011), arXiv:1012.4220.

[38] R. S. K. Mong, D. J. Clarke, J. Alicea, N. H. Lindner, P. Fendley, C. Nayak, Y. Oreg, A. Stern, E. Berg, K. Shtengel, et al. (2013), to appear in Phys. Rev. X, arXiv:1307.4403.

[39] B. I. Halperin, A. Stern, and S. M. Girvin, Phys. Rev. B 67, 
235313 (2003), cond-mat/0301442.

[40] J. Nilsson, A. R. Akhmerov, and C. W. J. Beenakker, Phys. Rev. Lett. 101, 120403 (2008).

[41] L. G. Herrmann, F. Portier, P. Roche, A. L. Yeyati, T. Kontos, and C. Strunk, Phys. Rev. Lett. 104, 026801 (2010), arXiv:0909.3243.

[42] A. Das, Y. Ronen, M. Heiblum, D. Mahalu, A. V. Kretinin, and H. Shtrikman, Nat. Commun. 3, 1165 (2012).

[43] Andreev conversion is essentially a chiral analogue of crossed Andreev reflection [40.42].

[44] Actually, the neutral sector exhibits trivial boundary conditions at the trench which allows one to eliminate $\mathcal{O}_{n}$ as well in Eq. 4. Hence $\Delta_{n}=0$, implying instability of the Andreev fixed point when $\nu>2$ (where the normal fixed point is stable).

\section{SUPPLEMENTARY INFORMATION}

In this section we characterize the instabilities leading to parafermionic zero-modes in a spin-unpolarized $\nu=2 / 3$ quantum Hall system, which for reasons elucidated below is a particularly promising platform for the transport anomalies described in the main text. To begin we summarize the edge theory for this quantum Hall state using $K$-matrix formalism [S1]. The edge modes of the unpolarized $2 / 3$ state are described by two bosonic fields $\phi_{\uparrow, \downarrow}$ and an associated $K$ matrix $\mathbf{K}=\left(\begin{array}{ll}1 & 2 \\ 2 & 1\end{array}\right)$ and charge vector $\mathbf{q}=\left(\begin{array}{l}1 \\ 1\end{array}\right)$. In this description, the edge electron density is given by $(1 / 2 \pi) \mathbf{q}^{\top} \partial_{x} \boldsymbol{\phi}$ and the filling fraction reads $\nu=\mathbf{q}^{\top} \mathbf{K}^{-1} \mathbf{q}=2 / 3$. A trench etched in the quantum Hall liquid brings two counterpropagating sets of such modes into close proximity with one another. Disregarding tunneling and pairing terms for the moment, the Lagrangian density for the edge modes opposite the trench is

$$
\mathcal{L}_{0}=\frac{1}{4 \pi} \partial_{x} \phi_{I}\left(\mathcal{K}_{I J} \partial_{t} \phi_{J}-V_{I J} \partial_{x} \phi_{J}\right),
$$

where we have four bosonic fields $\phi_{I}$, two for each side of the trench. The extended $K$-matrix for this doubled system is $\mathcal{K}=\left(\begin{array}{cc}\mathbf{K} & 0 \\ 0 & -\mathbf{K}\end{array}\right)$, while the matrix $\mathbf{V}$ captures the density-density interactions both within each edge and across the trench.

For simplicity, we assume the two edges are symmetric and that the interactions are invariant under $\mathrm{SU}(2)$ spin rotations. (Note that an equivalent SU(2) symmetry emerges due to disorder in the polarized $\nu=2 / 3$ state [S2]; here, however, the $\mathrm{SU}(2)$ symmetry is manifestly that of spin.) This gives us the generic for $\mathrm{m}$

$$
\mathbf{V}=\left(\begin{array}{llll}
v_{1} & v_{2} & v_{3} & v_{4} \\
v_{2} & v_{1} & v_{4} & v_{3} \\
v_{3} & v_{4} & v_{1} & v_{2} \\
v_{4} & v_{3} & v_{2} & v_{1}
\end{array}\right)
$$

Of course the SU(2) spin symmetry is not guaranteed microscopically. In particular, this symmetry is broken by a Zeeman field, which takes the form $H_{\text {Zeeman }}=(h / 2 \pi) \mathbf{n}_{\mathrm{t}}^{\top} \partial_{x} \boldsymbol{\phi}$, with $\mathbf{n}_{\mathrm{t}}^{\top}=(1,-1,1,-1)$. This term has scaling dimension 1 , but can be absorbed into $\mathcal{L}_{0}$ via a redefinition $\phi \rightarrow$ $\phi-h x \mathbf{V}^{-1} \mathbf{n}_{\mathrm{t}}$. However, this absorbtion will cause other terms involving $\exp \left(i \mathbf{n}_{\mathrm{t}}^{\top} \boldsymbol{\phi}\right)$ to oscillate on the length scale $\pi\left(v_{1}-v_{2}+v_{3}-v_{4}\right) / 2 h$.

Next we classify perturbations to Eq. (S1p that can generate instabilities. There are six types of gap-opening perturbations that are marginal when $h=0$ and density-density interactions between the two edges are absent, i.e. $v_{3}=v_{4}=0$. Charge hopping and pairing terms each come in two varieties due to spin degeneracy. We can divide these into perturbations that form either spin singlets or spin triplets across the trench. In addition, there are marginal perturbations that do not transfer charge but involve singlet or triplet spin correlations across the trench. These perturbations are listed in Table I along with their scaling dimensions (expressed in terms of parameters $u$ and $v$ defined in the caption).

\begin{tabular}{|l|l|l|}
\hline Process & Operators & Scaling Dimension \\
\hline singlet hopping & $e^{ \pm \frac{1}{2} \mathbf{c}_{\mathrm{h}}^{\top} \mathcal{K} \phi} e^{ \pm \frac{1}{2} \mathbf{n}_{\mathrm{s}}^{\top} \mathcal{K} \phi}$ & $\frac{1}{2} e^{2 u}+\frac{3}{2} e^{2 v}$ \\
triplet hopping & $e^{ \pm \frac{1}{2} \mathbf{c}_{\mathrm{h}}^{\top} \mathcal{K} \phi} e^{ \pm \frac{1}{2} \mathbf{n}_{\mathrm{t}}^{\top} \mathcal{K} \phi}$ & $\frac{1}{2} e^{-2 u}+\frac{3}{2} e^{2 v}$ \\
singlet pairing & $e^{ \pm \frac{1}{2} \mathbf{c}_{\mathrm{p}}^{\top} \mathcal{K} \phi} e^{ \pm \frac{1}{2} \mathbf{n}_{\mathrm{s}}^{\top} \mathcal{K} \phi}$ & $\frac{1}{2} e^{2 u}+\frac{3}{2} e^{-2 v}$ \\
triplet pairing & $e^{ \pm \frac{1}{2} \mathbf{c}_{\mathrm{p}}^{\top} \mathcal{K} \phi} e^{ \pm \frac{1}{2} \mathbf{n}_{\mathrm{t}}^{\top} \mathcal{K} \phi}$ & $\frac{1}{2} e^{-2 u}+\frac{3}{2} e^{-2 v}$ \\
neutral singlet & $e^{ \pm \mathbf{n}_{\mathrm{s}}^{\top} \mathcal{K} \phi}$ & $2 e^{2 u}$ \\
neutral triplet & $e^{ \pm \mathbf{n}_{\mathrm{t}}^{\top} \mathcal{K} \phi}$ & $2 e^{-2 u}$ \\
\hline
\end{tabular}

TABLE I: The six types of gap-opening perturbations that are marginal when $h=v_{3}=v_{4}=0$. There are two representatives of each neutral type and four of each charged type. Here $\mathbf{c}_{\mathrm{h}}^{\top}=(1,1,-1,-1), \mathbf{c}_{\mathrm{p}}^{\top}=(1,1,1,1), \mathbf{n}_{\mathrm{s}}^{\top}=(1,-1,-1,1)$, and $\mathbf{n}_{\mathrm{t}}^{\top}=(1,-1,1,-1)$. The important parameters $u$ and $v$ are defined as $\tanh 2 u=-\frac{\left(v_{3}-v_{4}\right)}{v_{1}-v_{2}}$ and $\tanh 2 v=-\frac{\left(v_{3}+v_{4}\right)}{v_{1}+v_{2}}$.

We now focus our attention on a system in which an ordinary $s$-wave superconductor couples to the trench, and therefore proximity-induces singlet pairing. Likewise, we will assume that electron hopping across the trench acts merely to restore the original quantum Hall state, rather than introducing additional spin flips. We therefore choose parameters such that singlet pairing, singlet hopping, and neutral singlet coupling are the dominant perturbations. Note that these terms are unaffected by the Zeeman field, while the triplet terms will have oscillating coefficients. Using Table I, we can find a region of parameter space for which all three singlet terms are simultaneously relevant by simply setting $u<0, v \approx 0$. This is reasonable as a physical regime, given that we must have $\left|v_{1}\right|>\left|v_{2}\right|$ for stability of the individual edges and repulsive interactions will generically give $v_{2}>0$, since $v_{2}$ is the density-density interaction between charges on the same edge. Likewise $v_{3}$ represents repulsion between charges of the same spin on opposite edges, and $v_{4}$ encodes repulsion between charges of opposite spins on opposite edges. It is not unreasonable to expect $v_{3}>v_{4}$, and thus $u<0$. We shall work in this parameter regime for the remainder of this analysis.

The fact that singlet pairing and direct tunneling across the 
trench can become simultaneously relevant for the same set of (reasonable) parameters is a great virtue of the unpolarized $\nu=2 / 3$ state. That is, the superconductor can induce a gap along the trench under the same conditions in which the $2 / 3$ state itself would also reform. This feature is difficult to achieve in other Abelian quantum Hall states. [Consider, e.g., the $\nu=2 / 5$ state with the same interaction matrix in Eq. (\$2). Here the scaling dimensions for the superconducting and tunneling perturbations respectively read $\Delta_{S}=\frac{1}{2} e^{2 u}+\frac{5}{2} e^{2 v}$ and $\Delta_{T}=\frac{1}{2} e^{2 u}+\frac{5}{2} e^{-2 v}$. These two terms cannot both be made relevant (i.e. $\Delta_{S, T}<2$ ) for the same values of $u$ and $v$.]

Crucially, although both singlet pairing and hopping terms are relevant at $\nu=2 / 3$, the gaps favored by these two processes are incompatible with one another. Rather, in any given region of the trench either the pairing or the hopping mechanism must win out in order for a gap to open. This is because the (charge-sector) fields that these two types of coupling try to pin are dual to one another. If one of them is pinned, the other must fluctuate. For $\nu=2 / 3$, though, there is no such issue in the neutral sector-i.e., all three singlet terms in Table 1 favor gapping the neutral fields in compatible ways. The neutral sector thus gaps out trivially, independent of which mechanism wins out.

As is the case when there is only one type of (fractional) edge mode, the boundary between a region of the trench gapped by pairing and one gapped by direct hopping supports a parafermionic zero-energy mode [S3-S5]. In this case, the zero mode has a $\mathbb{Z}_{3}$ character. The main text describes a number of nontrivial consequences for transport when these (and other types of zero modes) are probed with quantum Hall edges. As an aside, we also comment that the unpolarized $2 / 3$ case may be of particular interest for more traditional topological quantum information applications because of the complete gapping of the neutral sector. This gap partially inoculates the zero modes here against noise. In particular, stray electrons cannot directly affect the state of quantum information encoded in these zero modes, because they possess neither the correct charge $(2 e / 3,4 e / 3$, or $0 \bmod 2 e)$ nor spin (always 0) to do so.

[S1] X. G. Wen and A. Zee, Phys. Rev. B 46, 2290 (1992).

[S2] C. L. Kane, M. P. A. Fisher, and J. Polchinski, Phys. Rev. Lett. 72, 4129 (1994), cond-mat/9402108.

[S3] D. J. Clarke, J. Alicea, and K. Shtengel, Nat. Commun. 4, 1348 (2013), arXiv:1204.5479.

[S4] N. H. Lindner, E. Berg, G. Refael, and A. Stern, Phys. Rev. X 2, 041002 (2012), arXiv:1204.5733.

[S5] M. Cheng, Phys. Rev. B 86, 195126 (2012), arXiv:1204.6084. 